\title{
CONTROL AND OPERATIONAL STRATEGIES FOR AUTOMATIC EXCAVATION
}

D.A. Bradley, D.W. Seward, R.H. Bracewell, R.V. Chaplin and M.B. Widden

Department of Engineering,

University of Lancaster,

Lancaster, LAI 4YR, UK.

\begin{abstract}
The automation of the excavation process requires consideration not only of the design and operation of the excavator drive and control systems but also of the mechanics of the excavation process itself. The paper therefore discusses the developments necessary in these areas in relation to the operation of a simple back-hoe arm. In particular, consideration is given to the ability of a typical excavator to apply a force over its operating envelope, to the design and operation of the hydraulic system, to the modes of operation available for the arm and to the need to gather information on the excavation process itself. In addition, the operation of an automatic excavator in relation to the overall site is examined and consideration given to the need for the provision and supply of information in the context of the site.
\end{abstract}

1. Introduction

The introduction of robotic technologies into the Civil and Construction industries has been recognised (1) as providing a number of advantages, including:

- Improved quality and consistency of operation.

- Increased productivity from individual items of plant.

- Results independent of operator skills.

- Removal of site personnel from hazardous environments.

- Easier and more effective management of both plant and site.

- Better and more effective planning of work sequences.

- Increased independance of plant operation with reduced support required from surveyors and banksmen

Within this framework, the automation of the excavation process can be considered at a number of levels ranging from the provision of operator support systems which retain the 'man-in-the-loop' to highly autonomous systems capable of the independent execution of a wide range of tasks. 
Integral to the development of a robotic excavator is the need for a greater understanding of the excavation process and the production of effective, low cost, computer controlled hydraulic systems. The research programme described is concerned with providing the increased understanding required and on the incorporation of this knowledge into the control and operational strategies for a robotic excavator $(2,3)$.

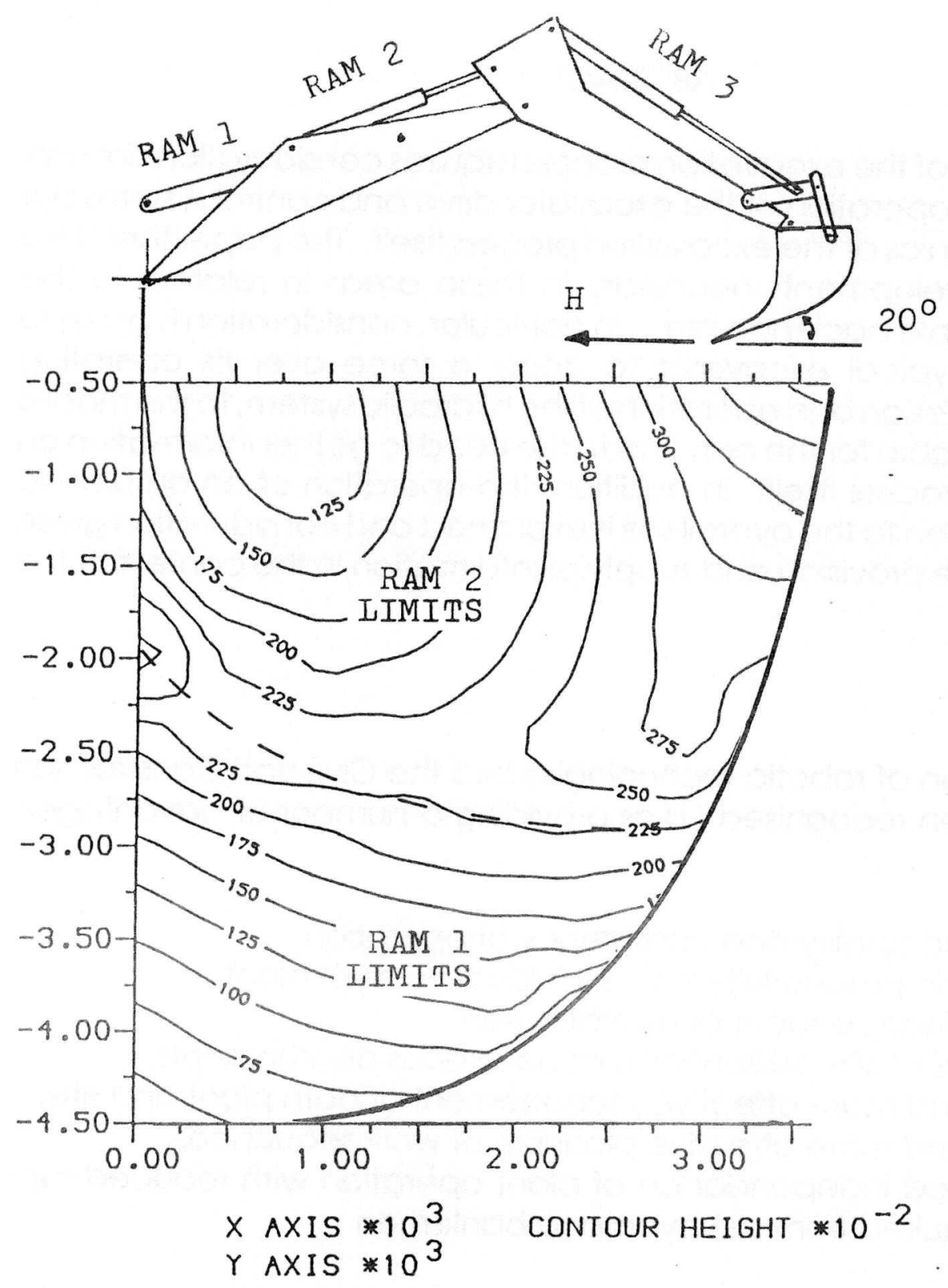

Figure 1: Horizontal forces exerted during excavation. 


\section{The Excavation Process}

The ability of an excavator to remove earth is related to its capacity to exert a force on the ground. Figures 1 and 2 illustrate the force profiles for a typical excavator in the vertical and horizontal directions respectively. From these figures it is apparent that the excavator is capable of exerting the greatest forces relatively near to the surface of the ground. However, in practice, the resistance of the ground to penetration by the bucket and subsequent removal is likely to increase with depth due to the effects of compaction.

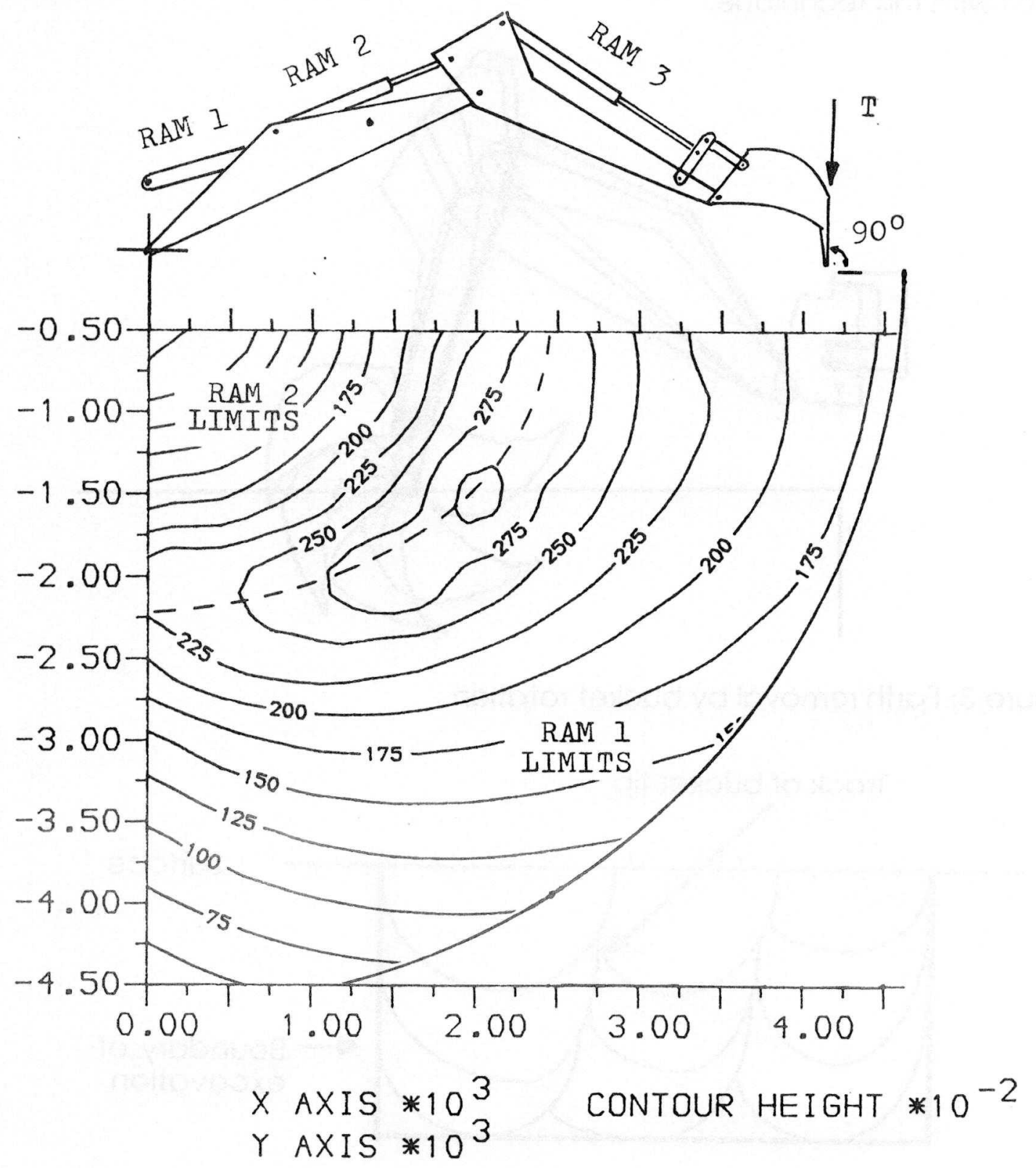

Figure 2: Vertical forces exerted during excavation. 
In relation to excavation it has been suggested (4) that the most effective approach to earth removal was one in which the bucket was first forced vertically into the ground after which it was filled with earth by a simple rotary action. This technique results in a pattern of earth removal as shown in figure 3 with a corresponding bucket track as shown by figure 4. This approach is, however, limited by the ability of the excavator to exert the required forces on the ground to achieve the necessary penetration. In addition, this technique suffers from the disadvantage with a manually operated machine that the bucket is hidden from the operator throughout the greater part of the excavation cycle. It is also not possible to achieve rapid cycle times with this technique.

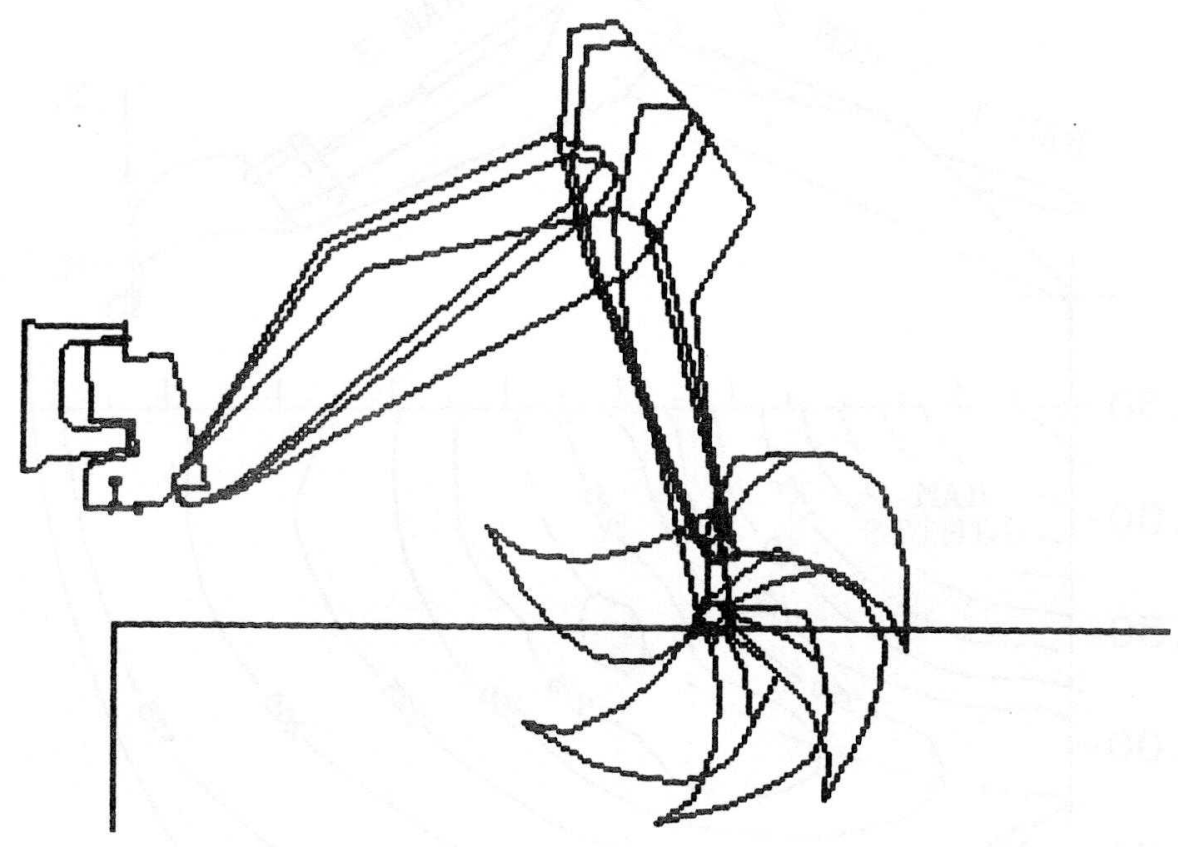

Figure 3: Earth removal by bucket rotation.

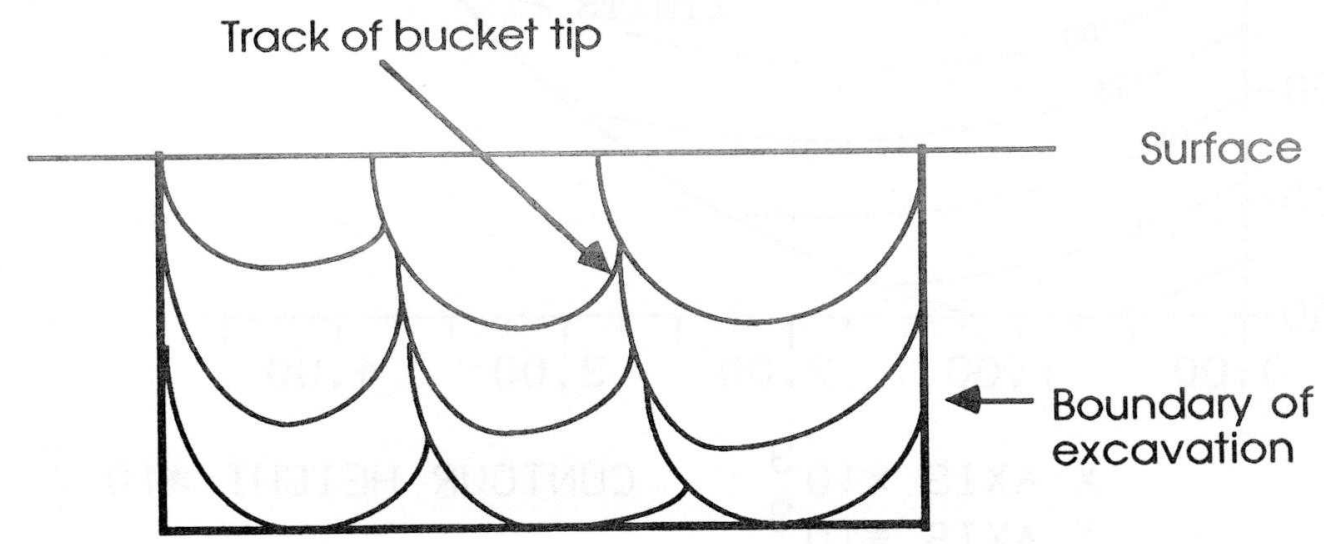

Figure 4: Bucket track for earth removal by bucket rotation. 
Investigation of the way in which experienced operators remove earth has suggested that an approach in which the ground is penetrated at a slight angle and then dragged towards the excavator to remove earth in 'slices' as in figure 5 is more effective. The track of the bucket is then as illustrated in figure 6 where the aim is to keep the bucket moving with a smooth and continuous motion. In this case, the soil is removed by a ploughing action and the depth of cut is determined by two factors:

1) The need to have the bucket full at the end of the stroke

2) The requirement that the excavating force is kept down to a sufficiently low value so as not to unduly impede the progress of the bucket, with shallower cuts obviously requiring less force.

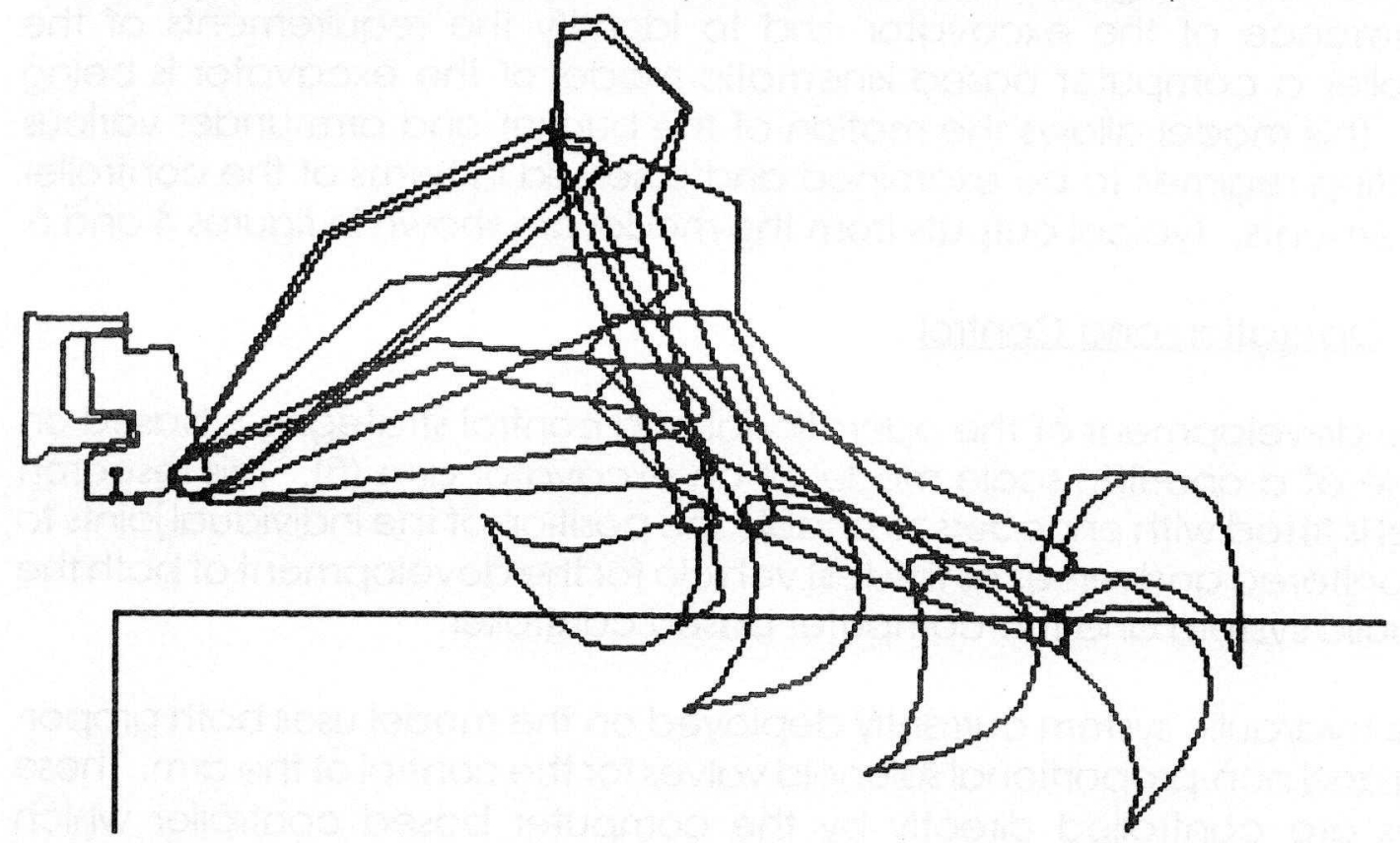

Figure 5: Earth removal by 'dragging' the excavator bucket.

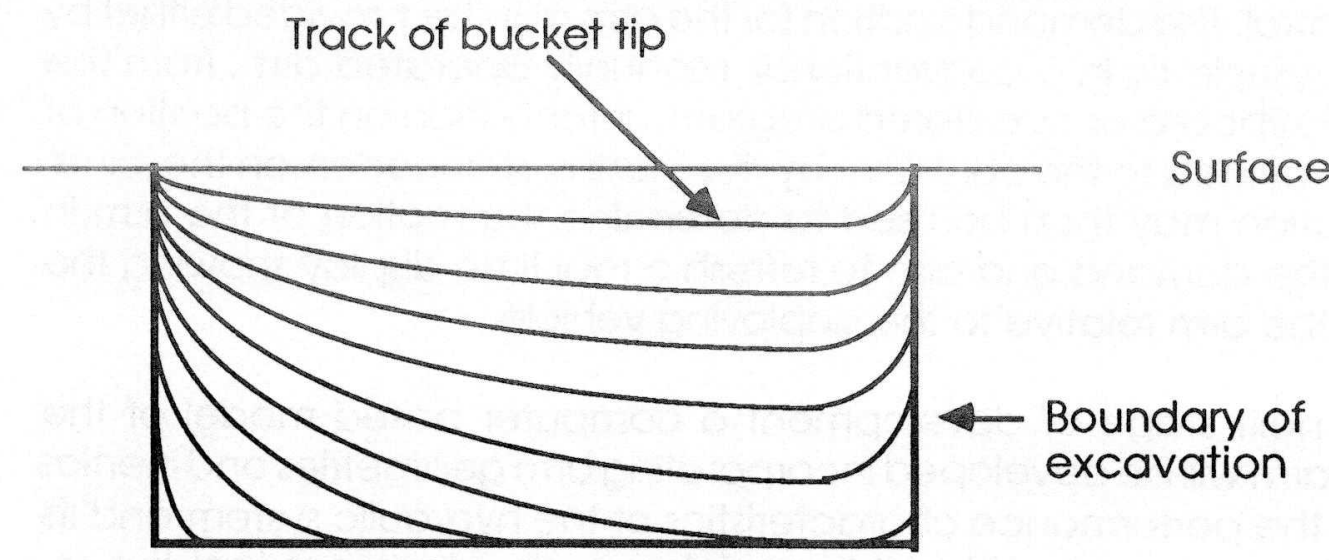

Figure 6: Bucket track for earth removal by 'dragging' the excavator bucket 
As the depth of an excavation increases, these two factors often come into conflict as the soil becomes harder and the capacity of the excavator to exert a force becomes less. More complex strategies such as those involving multiple passes to fill the bucket may then become appropriate.

Work is currently in progress at Lancaster to investigate the relationship between depth of cut, bucket angle and excavation force for various types of soil.

\section{Modelling}

In order to investigate the effect of individual operational strategies on the performance of the excavator and to identify the requirements of the controller a computer based kinematic model of the excavator is being used. This model allows the motion of the bucket and arm under various operating regimes to be examined and assessed in terms of the controller requirements. Typical outputs from the model are shown in figures 4 and 6 .

\section{Operation and Control}

The development of the operational and control strategies is based on the use of a one-fifth scale model of an excavator arm (5). This research model is fitted with encoders to enable the position of the individual joints to be monitored and serves as the test vehicle for the development of both the hydraulic system and the computer based controller.

The hydraulic system currently deployed on the model uses both proportional and non-proportional solenoid valves for the control of the arm. These valves are controlled directly by the computer based controller which modulates them in accordance with the demand to provide the required position control. The demand position for the arm may be provided either by means of joysticks as in a conventional, manually operated arm, from the computer keyboard or as a stored program. Information on the position of the arm is provided to the controller by the means of encoders on the joints. This information may then be used to determine the motion of the arm in relation to the demand and also to refresh a real time display showing the position of the arm relative to the deploying vehicle.

In the next stage of development a computer based model of the excavator arm will be developed incorporating arm geometries and inertias along with the performance characteristics of the hydraulic system and its associated components. Using this model as a development tool the required algorithms for a range of operational strategies including constant 
level control, teach and learn and obstacle avoidance would be developed and evaluated prior to testing on model arm referred to above. The investigation would also include a study of the effects of introducing novel hydraulic components, circuit arrangements and arm geometries upon the overall performance envelope.

The work on the control of the arm would be paralleled by an investigation into the associated sensing requirements for effective operation of the arm. This would include the development of techniques for the location of the arm relative to the site - global positioning - and relative to the task - local positioning as well as sensors to record vehicle attitude during the digging cycle and for obstacle avoidance. This latter is of particular importance where buried pipes and cables may be encountered, for example, in the U.K. there are currently over 70,000 recorded instances of damage to buried services during excavation and the introduction and adoption of robotic excavation must to some degree be dependent on the ability to reduce this figure.

\subsection{Operational strategies}

A variety of operational strategies of increasing complexity are under consideration with, initially, the emphasis on the provision of operator support functions rather than autonomous operation. By this means a sequential, staged development towards fully autonomous operation is envisaged with the advantage that applicable, stand alone sub-functions should become available relatively early in the programme. These sub-functions would then be incorporated within the full control package at a later date.

The staged development programme initially envisages the introduction of a 'dig to level' feature enabling an operator to achieve a controlled level without the need for outside assistance such as that provided by a banksman. The level would be set either by the operator or by reference to some external, task specific, reference framework such as a laser line. The provision of this feature would then provide the basis for a range of further developments incorporating information gathered from a site studies programme and leading ultimately to the provision of an expert system based controller for the excavation process.

\section{5. $\quad$ Site Studies}

In order to establish the necessary knowledge base for the development of an expert system for the excavation a programme of site studies is being undertaken to establish the nature of the excavation process. This will involve visiting a number of sites of differing characteristics to monitor current 
practices as part of the process of knowledge elicitation. The information gathered from these site studies will be combined with the developments in hydraulic systems and operational data obtained from the laboratory based research model for implementation in the final system.

\section{Discussion and Conclusions}

The achievement of an effective system of robotic excavation will require a significant programme of research over the next few years. In particular, developments are required in the areas of:

- The improved understanding and definition of the excavation process at both the tactical and strategic levels.

- The development of effective control systems capable of operating autonomously in an unstructured environment.

- The provision of appropriate sensors and site communications systems.

The research programme at Lancaster is aimed at providing the answers to some of these problems.

\section{References}

1 CIRIA (UK), 'Feasibility Study on the Application of Advanced Robotics to the Civil and Construction Industry', Advanced Robotics Initiative Feasibility Study, July 1987.

2 Whittaker, W., et al., 'First Results in Automated Pipe Excavation', 2nd Conference on Robotics in Construction, Carnegie Mellon University, USA, June 1985

3 Sakai, T. \& Cho, K., 'Operational System for Hydraulic Excavator for Deep Trench Works', 5th International Symposium on Robotics in Construction, Tokyo, Japan, June 1988,p709-716

4 Zenelin, A.N., Balovnev, V.I. \& Kerov, I.P., 'Machines for Moving the Earth', A.A. Balkema (Pub), Rotterdam, 1987

5 Seward, D., Bradley, D.A. \& Bracewell, R.H., 'Research Models for Automatic Excavation', 5th International Symposium on Robotics in Construction, Tokyo, Japan, June 1988,p703-709 